\title{
Relativistic Bound States of Spinless Particle by the Cornell Potential Model in External Fields
}

\author{
Sameer M. Ikhdair ${ }^{1,2}$ \\ ${ }^{1}$ Department of Physics, Faculty of Science, An-Najah National University, Nablus, West Bank, Palestine \\ ${ }^{2}$ Department of Electrical and Electronic Engineering, Near East University, 922022 Nicosia, Northern Cyprus, Turkey \\ Correspondence should be addressed to Sameer M. Ikhdair; sameer.ikhdair@najah.edu
}

Received 7 May 2013; Accepted 4 August 2013

Academic Editor: Ira Rothstein

Copyright (C) 2013 Sameer M. Ikhdair. This is an open access article distributed under the Creative Commons Attribution License, which permits unrestricted use, distribution, and reproduction in any medium, provided the original work is properly cited.

\begin{abstract}
The two-dimensional (2D) relativistic bound states of a spinless particle placed in scalar $S(r)$ and vector $V(r)$ Cornell potentials (with $S(r)>V(r)$ ) are obtained under the influence of external magnetic and Aharonov-Bohm (AB) flux fields using the wave function ansatz method. The relativistic energy eigenvalues and wave functions are found for any arbitrary state with principal $n$ and magnetic $m$ quantum numbers. Further, we obtain the eigensolutions in any dimensional space $D$ without external fields. We also find the relativistic and nonrelativistic bound states for Coulomb, harmonic oscillator, and Kratzer potentials.
\end{abstract}

\section{Introduction}

Relativistic wave equations such as Dirac and Klein-Gordon equations have been of much concern for theoretical physicists $[1,2]$. The KG is almost employed in describing the spinzero particle dynamics in relativistic quantum mechanics. The existence of strong fields or high speeds introduces relativistic phenomena that cannot be described using the Schrödinger equation. A discussion of the relativistic behavior of spin-zero particles needs understanding the single particle spectrum and consequently the exact solutions to the KG which are constructed using the four-vector potential $A_{\nu}(\nu=0,1,2,3)$ and the scalar potential $S(r)$. It can be said that the motion of a relativistic spin-zero particle in a potentials is described by the KG equation with the potential $S(r)$ and $V(r)[3]$.

Over the past few years, there has been an increasing interest in obtaining the exact solutions to the spinless KG particles exposed to different potential models [4-6]. Various techniques are used in solving such wave equations as the Nikiforov-Uvarov (NU) method [7, 8], the supersymmetric approach [9-11], the point canonical method [12, 13], the asymptotic iteration method $[14,15]$, the exact quantization rule $[16,17]$, the shifted $1 / N$ expansion technique [18], parametrized version of NU method [19], perturbative treatment [20], and wave function ansatz method [21, 22].
One of the potentials that has received much attention in particle physics was the Cornell (Coulomb plus linear) potential. It has been used successfully in models describing binding states of heavy quarks [23-25]. The Cornell potential contains a short range part dominated by a Coulombicterm of quark and gluon interaction $\sim \alpha_{s} / r$ from perturbative quark chromodynamics (QCD) and the large distance quark confinement known from lattice $\mathrm{QCD}$ as a linear term $\sim r$ [26-31]. In some situations when the parameter $b$ is small, it provides a particular case of perturbed Coulomb problem in atomic physics [32]. This potential has been used to study the strange, charmed, and beautiful baryon masses in the framework of variational approach [33].

The ground state energy $(n=0)$ of the KG equation with scalar-vector Killingbeck potentials [34] has been found using the ansatz method [35-37]. Furthermore, the ground state energy of the KG equation with scalar-vector Cornell potentials [38] has been calculated under the influence of the magnetic and Aharonov-Bohm fields.

Very recently, we have studied the scalar charged particle in scalar-vector (harmonic oscillator plus Cornell) potentials with and without external magnetic and Aharonov-Bohm flux fields [39] and obtained its relativistic energy eigenvalues and wave functions using the analytical exact iteration method [40,41]. The behavior of a spinless relativistic particle moving under the Cornell potential with the Coulomb 
singularity in the presence of static magnetic and $A B$ flux fields has not been investigated yet for any principal $n$ and magnetic $m$ quantum numbers.

The aim of this work is to extend [38] to include the bound state solutions for any $n$ and $m$ quantum numbers. Thus we solve the radial KG equation in $2 \mathrm{D}$ space for unequal mixture of scalar-vector Cornell potentials with and without constant magnetic and $\mathrm{AB}$ flux fields for the first time. For the real relativistic bound state solutions, we should choose $S(r) \geq V(r)$ and $m_{e}<E$, where $m_{e}$ is the mass of particle (electron) and $E$ is its energy while the nonrelativistic solution can be obtained when $S(r)=V(r)$. We choose not to pursue the case $S(r)=-V(r)$ since its nonrelativistic limit is the trivial interaction-free mode. This does not diminish the importance of such problems. It only limits its contribution to the relativistic regime. This includes the general case $V(r)=$ $\beta S(r)$, where $|\beta| \leq 1$, where $\beta$ is a real number. On the other hand, the class of problems $S(r)=\beta V(r)$ with $|\beta|<1$ are unbounded as we will see from our solution. Moreover, we present the exact energy eigenvalues and wave functions of the pure scalar Cornell potential in presence of magnetic field with various values of Larmor frequencies for any vibrational $n$ and rotational $m$ quantum numbers using the wave function ansatz and series method [40-44]. In addition, some special cases of KG solutions are obtained for the Coulomb and harmonic oscillator interactions in $3 \mathrm{D}$ space without the external fields.

The structure of this paper is as follows. In Section 2, we introduce the KG equation in $2 \mathrm{D}$ space for unequal mixture of scalar-vector Cornell potential under external magnetic and $\mathrm{AB}$ flux fields. We obtain the energy eigenvalues and wave functions for any $n$ and $m$ state. Section 3 is devoted for discussing some particular cases such as KG-scalar Cornell problem with and without external fields, KG-Coulomb, and KG-harmonic oscillator problems without external fields in any dimensional space. Our concluding remarks are given in Section 3.

\section{2D Relativistic Bound State Solutions in External Fields}

The Klein-Gordon atom for the spinless particle with mass $m_{e}$ and charge $-e$ moving in external electromagnetic field and $\mathrm{AB}$ flux field given by potentials $V(r), S(r)$, and $\vec{A}$ reads $[45,46]$

$$
\begin{aligned}
& {\left[c^{2}\left(\vec{p}+\frac{e}{c} \vec{A}\right)^{2}-(E-V(r))^{2}\right.} \\
& \left.\quad+\left(m_{e} c^{2}+S(r)\right)^{2}\right] \psi(r, \phi)=0 .
\end{aligned}
$$

We choose [47-52]

$$
\vec{A}=\frac{1}{2} \vec{B} \times \vec{r}+\frac{\Phi_{\mathrm{AB}}}{2 \pi r} \widehat{\phi}
$$

and the scalar-vector potentials in the form of the Cornell (Coulomb plus linear) potentials describing quark-antiquark interactions [23, 24, 28-31] are as follows:

$$
V(r)=-\frac{a_{v}}{r}+b_{v} r, \quad S(r)=-\frac{a_{s}}{r}+b_{s} r,
$$

which are extensively used in particle physics [30, 31]. Moreover, the vector potential in the symmetric gauge is defined by $\vec{A}=\vec{A}_{1}+\vec{A}_{2}$ such that $\vec{\nabla} \times \vec{A}_{1}=\vec{B}$ and $\vec{\nabla} \times \vec{A}_{2}=0$, where the applied magnetic field $\vec{B}=(0,0, B)$ is perpendicular to the plane of transversal motion of the particle and $\vec{A}_{2}$ describes the additional AB flux field $\Phi_{\mathrm{AB}}$ created by a solenoid in cylindrical coordinates [50-52]. The wave function in (1) is defined by

$$
\psi(r, \phi)=\frac{1}{\sqrt{2 \pi}} e^{i m \phi} \frac{R(r)}{\sqrt{r}}, \quad m=0, \pm 1, \pm 2, \ldots
$$

where $m$ is the eigenvalue of angular momentum. Now in the solution of the KG equation the relationship between the attractive scalar and repulsive vector potentials is given by $V(r)=\beta S(r)$, where $|\beta| \leq 1$ is arbitrary constant that refers to the existence of energy eigenvalues and hence the KG equation could be reduced to the Schrödinger-type second order differential equation as

$$
\begin{aligned}
& {\left[c^{2}\left(\vec{p}+\frac{e}{c} \vec{A}\right)^{2}+2\left(E V(r)+m_{e} c^{2} S(r)\right)\right.} \\
& \left.+S^{2}(r)-V^{2}(r)+m_{e}^{2} c^{4}-E^{2}\right] \psi(r, \phi)=0 .
\end{aligned}
$$

In case when $S(r)=V(r)$, the above equation reduces to the Schrödinger equation. Now we will treat the energy solutions of the two cases in (5) as follows.

2.1. The Energy Solution. The energy states require that $S(r)=$ $V(r)$ (i.e., $\beta=1$ case) which, in the nonrelativistic limit, corresponds to the solution of the wave equation:

$$
\begin{aligned}
& \left\{\frac{1}{2 \mu}\left[-i \hbar \vec{\nabla}+\frac{e}{c}\left(\frac{B r}{2}+\frac{\Phi_{\mathrm{AB}}}{2 \pi r}\right) \hat{\phi}\right]^{2}+2 V_{K}(r)-E\right\} \\
& \times \psi(r, \phi)=0,
\end{aligned}
$$

where $\psi(r, \phi)$ stands for nonrelativistic wave function. Thus, the choice of the case $S(r)=V(r), \beta=1$ is not mostly to do with finding exact analytical solution but simply to enable one to obtain a non-relativistic limit with a potential function $2 V(r)$ and not $V(r)$. Accordingly, it would be natural to scale the potential term in (6) so that in the non-relativistic limit the interaction potential becomes $V(r)$ not $2 V(r)$. With the scalar $S(r)$ and vector $V(r)$ Cornell potentials: given in (3), we can rewrite (5) as

$$
\begin{aligned}
& \left\{c^{2}\left[-i \hbar \vec{\nabla}+\frac{e}{c}\left(\frac{B r}{2}+\frac{\Phi_{\mathrm{AB}}}{2 \pi r}\right) \hat{\phi}\right]^{2}\right. \\
& \left.-\frac{w_{1}}{r^{2}}-\frac{w_{2}}{r}+w_{3}+w_{4} r+w_{5} r^{2}\right\} \psi(r, \phi)=0,
\end{aligned}
$$


with

$$
\begin{gathered}
w_{1}=a_{v}^{2}-a_{s}^{2}, \quad w_{2}=2\left(E a_{v}+m_{e} c^{2} a_{s}\right), \\
w_{3}=m_{e}^{2} c^{4}-E^{2}-2\left(a_{s} b_{s}-a_{v} b_{v}\right), \\
w_{4}=2\left(E b_{v}+m_{e} c^{2} b_{s}\right), \quad w_{5}=b_{s}^{2}-b_{v}^{2}, \\
\nabla^{2}=\frac{1}{\rho} \frac{\partial}{\partial \rho}+\frac{\partial^{2}}{\partial \rho^{2}}+\frac{1}{\rho^{2}} \frac{\partial^{2}}{\partial \phi^{2}}+\frac{\partial^{2}}{\partial z^{2}} .
\end{gathered}
$$

By inserting (4) and (9) into (7), we can obtain $[40,41,53]$

$$
\frac{d^{2} R(r)}{d r^{2}}+\left(\frac{C_{1}}{r^{2}}+\frac{C_{2}}{r}-C_{3}-C_{4} r-C_{5} r^{2}\right) R(r)=0,
$$

with

$$
\begin{gathered}
C_{1}=-m^{\prime 2}+\frac{1}{4}+\frac{a_{v}^{2}-a_{s}^{2}}{\hbar^{2} c^{2}}, \quad C_{2}=\frac{w_{2}}{\hbar^{2} c^{2}}, \\
C_{3}=\frac{2 m_{e} \omega_{L} m^{\prime}}{\hbar}+\frac{w_{3}}{\hbar^{2} c^{2}}, \\
C_{4}=\frac{w_{4}}{\hbar^{2} c^{2}}, \quad C_{5}=\frac{w_{5}}{\hbar^{2} c^{2}}+\left(\frac{m_{e} \omega_{L}}{\hbar}\right)^{2},
\end{gathered}
$$

and the asymptotic behaviors $R(0)=0$ and $R(\infty) \rightarrow 0$. Notice the energy depending on the strength of the magnetic field characterized by the Larmor frequency, $\omega_{L}$,

$$
\omega_{L}=\frac{\Omega}{2}, \quad \Omega=\frac{|e| B}{m_{e} c},
$$

and the new eigenvalue of angular momentum (magnetic quantum number), $m^{\prime}$,

$$
m^{\prime}=m+\xi, \quad \xi=\frac{\Phi_{\mathrm{AB}}}{\Phi_{0}}, m^{\prime}=1,2, \ldots
$$

where $\Phi_{0}=h c / e$ refers to the flux quantum and $\xi$ is an integer. The frequency $\Omega$ is called the cyclotron frequency of rotation [54] corresponding to the classical motion of a charged particle in a uniform magnetic field and $\Omega / 2$ is the Larmor frequency in units of $\mathrm{Hz}$ [54].

Hence, the Schrödinger-type equation with unequal scalar-vector Cornell potential can be written as

$$
\begin{aligned}
& \frac{d^{2} R(r)}{d r^{2}}+\frac{\left(E+m_{e} c^{2}\right)}{\hbar^{2} c^{2}} \\
& \quad \times\left[E-m_{e} c^{2}-U_{\text {eff }}\left(r, \omega_{L}, \xi\right)\right] R(r)=0,
\end{aligned}
$$

with the effective potential

$$
\begin{aligned}
U_{\text {eff }}\left(r, \omega_{L}, \xi\right) & \\
=\frac{1}{\left(E+m_{e} c^{2}\right)}[ & -\frac{2\left(E a_{v}+m_{e} c^{2} a_{s}\right)}{r} \\
& +2\left(E b_{v}+m_{e} c^{2} b_{s}\right) r \\
& +\left(m_{e}^{2} c^{2} \omega_{L}^{2}+b_{s}^{2}-b_{v}^{2}\right) r^{2} \\
& +\frac{\hbar^{2} c^{2}\left(m^{2}-1 / 4\right)+a_{s}^{2}-a_{v}^{2}}{r^{2}} \\
& \left.+2 \hbar \omega_{L} m_{e} c^{2} m^{\prime}\right] .
\end{aligned}
$$

It is interesting to look at the results obtained from (10) for a special case $C_{2}=C_{4}=0$; that is, we have KGpseudoharmonic problem:

$$
\frac{d^{2} R(r)}{d r^{2}}+\left(\frac{C_{1}}{r^{2}}-C_{5} r^{2}\right) R(r)=C_{3} R(r),
$$

which corresponds to the differential equation of a harmonic oscillator with a centrifugal term. It is similar form to the one given by (7) in [55] with the equivalence $C_{1} \rightarrow-l^{\prime}\left(l^{\prime}+1\right)$, $C_{5} \rightarrow \alpha^{2}$, and $C_{3} \rightarrow-\lambda_{n}(\alpha, l$, and $\lambda$ are the parameters used in [55]). So by putting the parameter values,

$$
\begin{gathered}
l^{\prime}= \pm \sqrt{m^{\prime 2}+\frac{a_{s}^{2}-a_{v}^{2}}{\hbar^{2} c^{2}}}-\frac{1}{2}, \\
\alpha=\frac{1}{\hbar} \sqrt{\frac{b_{s}^{2}-b_{v}^{2}}{c^{2}}+\left(m_{e} \omega_{L}\right)^{2}}, \\
\lambda_{n}=\frac{1}{\hbar^{2} c^{2}}\left[m_{e}^{2} c^{4}-E^{2}+d_{s}^{2}-d_{v}^{2}\right. \\
\left.+2\left(a_{v} b_{v}-a_{s} b_{s}+E d_{v}+m_{e} c^{2} d_{s}\right)\right] \\
-\frac{2 m_{e} \omega_{L} m^{\prime}}{\hbar},
\end{gathered}
$$

into (14) of [54], we obtain the energy equation as

$$
\begin{aligned}
E^{2}- & m_{e}^{2} c^{4}+2\left(a_{s} b_{s}-a_{v} b_{v}\right) \\
= & 2 m_{e} c^{2} \hbar \omega_{L} m^{\prime} \\
& +2 \hbar c^{2}\left(2 n+1+\sqrt{\left.m^{\prime 2}+\frac{a_{s}^{2}-a_{v}^{2}}{\hbar^{2} c^{2}}\right)}\right. \\
& \times \sqrt{\frac{b_{s}^{2}-b_{v}^{2}}{c^{2}}+\left(m_{e} \omega_{L}\right)^{2}}, \quad a_{s}>a_{v}, b_{s}>b_{v} .
\end{aligned}
$$

Now we find a solution to (10) by making the following choice of the wave function [40, 41]:

$$
R_{n m}(r)=\exp \left(\frac{1}{2} p r^{2}+q r\right) \sum_{n=0}^{\infty} a_{n} r^{n+\delta},
$$


where $p$ and $q$ are parameters whose values are to be determined in terms of the potential parameters. Substituting (19) into (10), we obtain the following series [40]:

$$
\begin{gathered}
\sum_{n=0}^{\infty} a_{n} S_{n} r^{n+\delta-2}+\sum_{n=1}^{\infty} a_{n-1} T_{n-1} r^{n+\delta-2} \\
+\sum_{n=2}^{\infty} a_{n-2} W_{n-2} r^{n+\delta-2}=0
\end{gathered}
$$

with

$$
\begin{gathered}
S_{n}=(n+\delta)(n+\delta-1)+C_{1}, \\
T_{n-1}=2 q(n+\delta-1)+C_{2}, \\
W_{n-2}=q^{2}+2 p\left(n+\delta-\frac{3}{2}\right)-C_{3}, \\
p= \pm \sqrt{\frac{1}{\hbar^{2} c^{2}}\left(b_{s}^{2}-b_{v}^{2}\right)+\left(\frac{m_{e} \omega_{L}}{\hbar}\right)^{2},} \\
p q=\frac{1}{\hbar^{2} c^{2}}\left(E b_{v}+m_{e} c^{2} b_{s}\right), \quad b_{s}>b_{v},
\end{gathered}
$$

where the two parameters $p$ and $q$ are determined in order that the radial wave functions $R_{n m}(r)$ must be finite everywhere and vanish at $r=0$ and as $r \rightarrow \infty$. In the absence of the magnetic field we require that $a_{s}>a_{v}$.

To obtain the recurrence relation which can connect various expansion coefficients $a_{n}$, we make identical powers of $r$ in (20), that is, equate the coefficients of $r^{n+\delta-2}$ to zero. Thus, the relations become

$$
\begin{gathered}
a_{0}\left[\delta(\delta-1)+C_{1}\right]=0 \Longrightarrow \delta^{2}+C_{1}=\delta, \quad a_{0} \neq 0, \\
\delta= \pm \sqrt{m^{\prime 2}+\frac{1}{\hbar^{2} c^{2}}\left(a_{s}^{2}-a_{v}^{2}\right)}+\frac{1}{2}, \quad \delta \neq 0 \Longrightarrow a_{s}>a_{v} \\
a_{1}=-\frac{\left(2 q \delta+C_{2}\right)}{2 \delta} a_{0}, \quad \delta \neq 0 \\
a_{2}^{\prime}=-\frac{\left[p(2 \delta+1)+q^{2}-C_{3}\right] a_{0}+\left[2 q(\delta+1)+C_{2}\right] a_{1}}{2(2 \delta+1)}, \\
a_{3}=-\frac{\left(2 p q-C_{4}\right) a_{0}+\left[p(2 \delta+3)+q^{2}-C_{3}\right] a_{1}}{6(\delta+1)} \\
+\frac{\left[2 q(\delta+2)+C_{2}\right] a_{2}}{6(\delta+1)},
\end{gathered}
$$

has been selected. The power series for large values of $\delta$ or $\mathrm{m}^{\prime}$ is convergent. For convenience, we take the ratio of two successive terms, that is, $a_{n+1} / a_{n}$, which becomes

$$
\begin{aligned}
\frac{a_{1}}{a_{0}}=- & \left(q+\frac{C_{2}}{2 \delta}\right) \longrightarrow-q \quad \text { when } \delta \rightarrow \infty, \\
\frac{a_{2}}{a_{0}}= & \frac{q^{2}\left(\delta+\left(C_{2} / 2 q\right)\right)\left[\delta+\left(C_{2} / 2 q\right)+1\right]}{\delta(2 \delta+1)} \\
& -\frac{\left[p(2 \delta+1)+q^{2}-C_{3}\right]}{2(2 \delta+1)} \\
& \longrightarrow \frac{q^{2}-p}{2} \quad \text { when } \delta \longrightarrow \infty .
\end{aligned}
$$

It is apparent from the above relations that the power series converges when $\delta \rightarrow \infty$. Hence, the series must be truncated (bounded) for $n=n_{\max }$. At this value of $n$, we obtain the following equations:

$$
p=-\sqrt{\frac{1}{\hbar^{2} c^{2}}\left(b_{s}^{2}-b_{v}^{2}\right)+\left(\frac{m_{e} \omega_{L}}{\hbar}\right)^{2}}, \quad p \neq 0,
$$

where the negative sign of the coefficient $p$ has been chosen in (22). Also the parameter $q$ in (22) is physically taken as

$$
q=-\frac{\left(E b_{v}+m_{e} c^{2} b_{s}\right)}{\hbar^{2} c^{2} \sqrt{\left(1 / \hbar^{2} c^{2}\right)\left(b_{s}^{2}-b_{v}^{2}\right)+\left(m_{e} \omega_{L} / \hbar\right)^{2}}}
$$

and the following relationship between the potential parameters must be achieved:

$$
\begin{aligned}
& \frac{E b_{v}+m_{e} c^{2} b_{s}}{\sqrt{\left(1 / \hbar^{2} c^{2}\right)\left(b_{s}^{2}-b_{v}^{2}\right)+\left(m_{e} \omega_{L} / \hbar\right)^{2}}} \\
& =\frac{E a_{v}+m_{e} c^{2} a_{s}}{\left(n+\sqrt{m^{\prime 2}+\left(1 / \hbar^{2} c^{2}\right)\left(a_{s}^{2}-a_{v}^{2}\right)}-(1 / 2)\right)} .
\end{aligned}
$$


The energy equation can be obtained via (23) as

$$
\begin{aligned}
& E^{2}- m_{e}^{2} c^{4}+2\left(a_{s} b_{s}-a_{v} b_{v}\right) \\
&= 2 m_{e} c^{2} \hbar \omega_{L} m^{\prime} \\
&+2 \hbar^{2} c^{2} \sqrt{\frac{\left(b_{s}^{2}-b_{v}^{2}\right)}{\hbar^{2} c^{2}}+\left(\frac{m_{e} \omega_{L}}{\hbar}\right)^{2}} \\
& \times\left(\sqrt{m^{\prime 2}+\frac{1}{\hbar^{2} c^{2}}\left(a_{s}^{2}-a_{v}^{2}\right)}+n-1\right) \\
&-\frac{\left(E a_{v}+m_{e} c^{2} a_{s}\right)^{2}}{\hbar^{2} c^{2}\left(n+\sqrt{m^{\prime 2}+\left(1 / \hbar^{2} c^{2}\right)\left(a_{s}^{2}-a_{v}^{2}\right)}-(1 / 2)\right)^{2}}, \\
& \quad n=0,1,2, \ldots . .
\end{aligned}
$$

We may find solutions to the above transcendental equation in terms of $m, n, \xi$, and $\omega_{L}$. The above energy equation is correct for $S>V$ and not only for $S=V$ (i.e, $\beta=1$ ). On the other hand, the solution for the case $S=-V$ can be obtained very simply from the above energy equation by making some transformations: $E \rightarrow-E$ and $V(r) \rightarrow-V(r)$, that is, $a_{v} \rightarrow-a_{v}, a_{s} \rightarrow-a_{s}, b_{v} \rightarrow-b_{v}$, and $b_{s} \rightarrow-b_{s}$ [39].

2.2. The Wave Function Solution. We can find the wave function in (4) with the help of (19), (24)-(28) as

$$
\begin{aligned}
\psi_{n, m}(r, \phi)= & C_{n, m} \frac{1}{\sqrt{2 \pi}} e^{i m \phi} r \sqrt{(m+\xi)^{2}+\left(1 / \hbar^{2} c^{2}\right)\left(a_{s}^{2}-a_{v}^{2}\right)} \\
& \times e^{-(1 / 2) \sqrt{\left(1 / \hbar^{2} c^{2}\right)\left(b_{s}^{2}-b_{v}^{2}\right)+\left(m_{e} \omega_{L} / \hbar\right)^{2}} r^{2}} \\
& \times e^{-\left(\left(E a_{v}+m_{e} c^{2} a_{s}\right) / \hbar^{2} c^{2}\left(n+\sqrt{m^{\prime 2}+\left(1 / \hbar^{2} c^{2}\right)\left(a_{s}^{2}-a_{v}^{2}\right)}-(1 / 2)\right)\right) r} \\
& \times \sum_{n=0}^{n_{\max }} a_{n} r^{n}
\end{aligned}
$$

with

$$
\begin{aligned}
a_{1}= & -a_{0} \\
& \times\left[-\frac{E b_{v}+m_{e} c^{2} b_{s}}{\hbar^{2} c^{2} \sqrt{\left(1 / \hbar^{2} c^{2}\right)\left(b_{s}^{2}-b_{v}^{2}\right)+\left(m_{e} \omega_{L} / \hbar\right)^{2}}}\right. \\
& \left.+\frac{E a_{v}+m_{e} c^{2} a_{s}}{\hbar^{2} c^{2}\left(\sqrt{m^{\prime 2}+\left(1 / \hbar^{2} c^{2}\right)\left(a_{s}^{2}-a_{v}^{2}\right)}+(1 / 2)\right)}\right],
\end{aligned}
$$

$$
\begin{aligned}
a_{2} & -a_{0} \frac{1}{2 \hbar^{2} c^{2}\left(2 \sqrt{m^{\prime 2}+\left(1 / \hbar^{2} c^{2}\right)\left(a_{s}^{2}-a_{v}^{2}\right)}+(3 / 2)\right)} \\
\times & \left\{\frac{\left(E a_{v}+m_{e} c^{2} a_{s}\right)^{2}}{\hbar^{2} c^{2}\left(n+\sqrt{m^{\prime 2}+\left(1 / \hbar^{2} c^{2}\right)\left(a_{s}^{2}-a_{v}^{2}\right)}-(1 / 2)\right)^{2}}\right. \\
& -\frac{2 \hbar^{2} c^{2}\left(\sqrt{m^{\prime 2}+\left(1 / \hbar^{2} c^{2}\right)\left(a_{s}^{2}-a_{v}^{2}\right)}+1\right)}{\sqrt{\left(1 / \hbar^{2} c^{2}\right)\left(b_{s}^{2}-b_{v}^{2}\right)+\left(m_{e} \omega_{L} / \hbar\right)^{2}}} \\
& +E^{2}-m_{e}^{2} c^{4}+2\left(a_{s} b_{s}-a_{v} b_{v}\right) \\
& -2 m_{e} c^{2} \hbar \omega_{L} m^{\prime}-\frac{2\left(E a_{v}+m_{e} c^{2} a_{s}\right)^{2}}{\hbar^{2} c^{2}} \\
& \times\left[1-\frac{\sqrt{m^{\prime 2}+\left(1 / \hbar^{2} c^{2}\right)\left(a_{s}^{2}-a_{v}^{2}\right)}+(1 / 2)}{\left(n+\sqrt{m^{\prime 2}+\left(1 / \hbar^{2} c^{2}\right)\left(a_{s}^{2}-a_{v}^{2}\right)}-(1 / 2)\right)}\right] \\
& {\left.\left[\frac{\sqrt{m^{\prime 2}+\left(1 / \hbar^{2} c^{2}\right)\left(a_{s}^{2}-a_{v}^{2}\right)}+(3 / 2)}{\left(n+\sqrt{m^{\prime 2}+\left(1 / \hbar^{2} c^{2}\right)\left(a_{s}^{2}-a_{v}^{2}\right)}-(1 / 2)\right)}\right]\right\} }
\end{aligned}
$$

where $C_{n, m}$ is the normalization constant.

Notice that the present model has been solved in $2 \mathrm{D}$ space with an external uniform magnetic field since it is perpendicular to the plane where the vector and scalar Cornell potentials have the dimensions of $r=\rho$ and $\phi$; that is, $V=V(\rho, \phi)$ [52]. However, without the magnetic field, the model can be solved in any desired dimensional space by considering the change $m \rightarrow l+(D-2) / 2[56,57]$, where $D$ refers to a spatial dimensional space and also the existence of interdimensional degeneracy.

\section{Some Special Cases}

In this section, we will obtain the energy levels with and without external magnetic field and $A B$ flux field and the energy levels in three dimensional (3D) space. Two special cases of much interest are considered, namely, the Coulomb and harmonic oscillator.

Firstly, let us consider the present system without external fields; that is, $\omega_{L}=\xi=0$. We are solving the Schrödinger-type equation with the effective potential:

$$
\begin{aligned}
& U_{\mathrm{eff}}(r)=\frac{1}{\left(E+m_{e} c^{2}\right)} \\
& \times\left[-\frac{2\left(E a_{v}+m_{e} c^{2} a_{s}\right)}{r}+2\left(E b_{v}+m_{e} c^{2} b_{s}\right) r\right. \\
& \left.+\left(b_{s}^{2}-b_{v}^{2}\right) r^{2}+\frac{\hbar^{2} c^{2}\left(m^{2}-1 / 4\right)+a_{s}^{2}-a_{v}^{2}}{r^{2}}\right] \text {. }
\end{aligned}
$$


Hence, the energy equation and wave functions reduce to

$$
\begin{gathered}
E_{n m}= \pm\left[m_{e}^{2} c^{4}-2 \gamma_{1}+2 \hbar c \gamma_{2}\left(\sqrt{m^{2}+\frac{\gamma_{3}}{\hbar^{2} c^{2}}}+n-1\right)\right. \\
\left.-\frac{\left(E a_{v}+m_{e} c^{2} a_{s}\right)^{2}}{\hbar^{2} c^{2}\left(n+\sqrt{m^{2}+\left(\gamma_{3} / \hbar^{2} c^{2}\right)}-(1 / 2)\right)^{2}}\right]^{1 / 2} \\
\gamma_{1}=a_{s} b_{s}-a_{v} b_{v}, \quad \gamma_{2}=\sqrt{b_{s}^{2}-b_{v}^{2}}, \\
\gamma_{3}=a_{s}^{2}-a_{v}^{2}, \quad b_{s}>a_{v}, \\
\psi_{n, m}(r, \phi) \\
=C_{n, m} \frac{1}{\sqrt{2 \pi}} e^{i m \phi} r \sqrt{m^{2}+\left(\gamma_{3} / \hbar^{2} c^{2}\right)} \\
\quad \times e^{-\left(\gamma_{2} / 2 \hbar c\right) r^{2}-\left(E a_{v}+m_{e} c^{2} a_{s}\right) / \hbar^{2} c^{2}\left(n+\sqrt{m^{2}+\left(\gamma_{3} / \hbar^{2} c^{2}\right)}-(1 / 2)\right) r} \\
\times \sum_{n=0}^{n_{\max }} a_{n} r^{n},
\end{gathered}
$$

where

$$
\begin{aligned}
a_{1}=-a_{0}\left[-\frac{E b_{v}+m_{e} c^{2} b_{s}}{\hbar c \sqrt{b_{s}^{2}-b_{v}^{2}}}\right. & \\
+ & \left.\frac{E a_{v}+m_{e} c^{2} a_{s}}{\hbar^{2} c^{2}\left(\sqrt{m^{2}+\left(1 / \hbar^{2} c^{2}\right)\left(a_{s}^{2}-a_{v}^{2}\right)}+(1 / 2)\right)}\right],
\end{aligned}
$$

$$
\begin{aligned}
a_{2}= & -a_{0} \frac{1}{2 \hbar^{2} c^{2}\left(2 \sqrt{m^{2}+\left(1 / \hbar^{2} c^{2}\right)\left(a_{s}^{2}-a_{v}^{2}\right)}+(3 / 2)\right)} \\
& \times\left\{E^{2}-m_{e}^{2} c^{4}+\left(\left(\left(E a_{v}+m_{e} c^{2} a_{s}\right)^{2}\right)\right.\right. \\
& \left.\times\left(\hbar^{2} c^{2}\left(n+\sqrt{m^{2}+\frac{1}{\hbar^{2} c^{2}}\left(a_{s}^{2}-a_{v}^{2}\right)}-\frac{1}{2}\right)^{2}\right)^{-1}\right) \\
& -\frac{2 \hbar^{2} c^{2}\left(\sqrt{m^{2}+\left(1 / \hbar^{2} c^{2}\right)\left(a_{s}^{2}-a_{v}^{2}\right)}+1\right)}{\sqrt{\left(1 / \hbar^{2} c^{2}\right)\left(b_{s}^{2}-b_{v}^{2}\right)}} \\
& +2\left(a_{s} b_{s}-a_{v} b_{v}\right)-\frac{2\left(E a_{v}+m_{e} c^{2} a_{s}\right)^{2}}{\hbar^{2} c^{2}}
\end{aligned}
$$

It is clear that, for the case $S(r) \geq V(r)$, there exist bound state solutions for a relativistic spin-zero particle. For $S(r)=$ $V(r)$, the KG equation reduces to a Schrödinger-like equation and thus the bound state solutions are easy to obtain using well-known methods developed in nonrelativistic quantum mechanics [45].

If the vector potential is taken zero, that is, $V(r)=0, a_{v}=$ $b_{v}=0$, the effective potential is

$$
\begin{aligned}
U_{\mathrm{eff}}(r)= & \frac{1}{\left(E+m_{e} c^{2}\right)} \\
& \times\left[-\frac{2 m_{e} c^{2} a_{s}}{r}+2 m_{e} c^{2} b_{s} r\right. \\
& \left.+b_{s}^{2} r^{2}+\frac{\hbar^{2} c^{2}\left(m^{2}-1 / 4\right)+a_{s}^{2}}{r^{2}}\right] .
\end{aligned}
$$

Therefore, the energy can be found as

$$
\begin{aligned}
E_{n m}= \pm & m_{e}^{2} c^{4}-2 a_{s} b_{s} \\
& -\frac{m_{e}^{2} c^{4} a_{s}^{2}}{\hbar^{2} c^{2}\left(n+\sqrt{m^{2}+\left(a_{s}^{2} / \hbar^{2} c^{2}\right)}-(1 / 2)\right)^{2}} \\
& \left.+2 \hbar c b_{s}\left(\sqrt{m^{2}+\frac{a_{s}^{2}}{\hbar^{2} c^{2}}}+n-1\right)\right]^{1 / 2}
\end{aligned}
$$

and wave function

$$
\begin{aligned}
\psi_{n, m}(r, \phi)= & C_{n, m} \frac{1}{\sqrt{2 \pi}} e^{i m \phi} r \sqrt{m^{2}+\left(a_{s}^{2} / \hbar^{2} c^{2}\right)} \\
& \times e^{-\left(b_{s} / 2 \hbar c\right) r^{2}-\left(m_{e} c^{2} a_{s} / \hbar^{2} c^{2}\left(n+\sqrt{m^{2}+\left(a_{s}^{2} / \hbar^{2} c^{2}\right)}-(1 / 2)\right)\right) r} \\
& \times \sum_{n=0}^{n_{\max }} a_{n} r^{n}
\end{aligned}
$$


Secondly, the energy equation and wave functions reduce to $3 \mathrm{D}$ space if we replace $m \rightarrow l+1 / 2$ as

$$
\begin{aligned}
E_{n l}= & \pm m_{e}^{2} c^{4}-2 a_{s} b_{s} \\
& -\frac{m_{e}^{2} c^{4} a_{s}^{2}}{\hbar^{2} c^{2}\left(n+\sqrt{(l+(1 / 2))^{2}+\left(a_{s}^{2} / \hbar^{2} c^{2}\right)}-(1 / 2)\right)^{2}} \\
& \left.+2 \hbar c b_{s}\left(\sqrt{\left(l+\frac{1}{2}\right)^{2}+\frac{a_{s}^{2}}{\hbar^{2} c^{2}}}+n-1\right)\right]^{1 / 2}
\end{aligned}
$$

$$
\begin{aligned}
& \psi_{n, l}(r, \phi) \\
& =C_{n, m} \frac{1}{\sqrt{2 \pi}} e^{i m \phi} r \sqrt{(l+1 / 2)^{2}+\left(a_{s}^{2} / \hbar^{2} c^{2}\right)} \\
& \\
& \quad \times e^{-\left(b_{s} / 2 \hbar c\right) r^{2}-\left(m_{e} c^{2} a_{s} / \hbar^{2} c^{2}\left(n+\sqrt{(l+1 / 2)^{2}+\left(a_{s}^{2} / \hbar^{2} c^{2}\right)}-(1 / 2)\right)\right) r} \\
& \quad \times \sum_{n=0}^{n_{\max }} a_{n} r^{n} .
\end{aligned}
$$

Thirdly, if the potential parameter $b_{s}=0$, the Cornell potential reduces to the relativistic Coulomb potential with $n=$ $n_{r}+1$. The effective potential becomes

$$
U_{\mathrm{eff}}(r)=\frac{1}{\left(E+m_{e} c^{2}\right)}\left[-\frac{2 m_{e} c^{2} a_{s}}{r}+\frac{\hbar^{2} c^{2} l(l+1)+a_{s}^{2}}{r^{2}}\right],
$$

which consists of the Coulomb plus new centrifugal term potentials. Therefore the energy levels and wave function become

$$
E_{n l}= \pm m_{e} c^{2}\left[1-\frac{a_{s}^{2}}{\hbar^{2} c^{2}\left(n_{r}+l+1\right)^{2}}\right]^{1 / 2}
$$

where

$$
\left(n_{r}+\sqrt{(l+1 / 2)^{2}+\frac{a_{s}^{2}}{\hbar^{2} c^{2}}}+\frac{1}{2}\right) \longrightarrow\left(n_{r}+l+1\right),
$$

is being obtained from (28). The wave function is

$$
\begin{aligned}
\psi_{n, l}(r, \phi)= & C_{n, m} \frac{1}{\sqrt{2 \pi}} e^{i m \phi} r^{l+1 / 2} \\
& \times e^{-\left(m_{e} c^{2} a_{s} / \hbar^{2} c^{2}\left(n_{r}+l+1\right)\right) r} \sum_{n=0}^{n_{\max }} a_{n} r^{n} .
\end{aligned}
$$

Thus, there exist solutions both for positive $E=+m_{e} c^{2}[1-$ $\left.\left(a_{s}^{2} /\left(\hbar^{2} c^{2}\left(n_{r}+l+1\right)^{2}\right)\right)\right]^{1 / 2}$ as well as for negative $E=-m_{e} c^{2}$ $\left[1-\left(a_{s}^{2} /\left(\hbar^{2} c^{2}\left(n_{r}+l+1\right)^{2}\right)\right)\right]^{1 / 2}$ energies, respectively. The solutions yielding negative energy are physically connected with antiparticles. Since antiparticles can indeed be observed in nature, we have already obtained an indication of the value of extending the nonrelativistic theory. In the nonrelativistic limit the difference of total energy $E$ of the particle and the rest mass $m_{e} c^{2}$ is small. Therefore, we define $E^{\prime}=E-m_{e} c^{2}$ and remark that the kinetic energy nonrelativistic which means $E^{\prime} \ll m_{e} c^{2}[45]$. In the nonrelativistic limit we obtain

$$
E_{n l}^{\prime}=-\frac{m_{e} a_{s}^{2}}{2 \hbar^{2}\left(n_{r}+l+1\right)^{2}} .
$$

Fourthly, if the potential parameter $b_{s}=0$, the Cornell potential reduces to the relativistic Kratzer potential $V(r)=$ $-D_{e}\left(\left(2 r_{e} / r\right)-\left(r_{e}^{2} / r^{2}\right)\right)$ with $n=n_{r}+1$. The effective potential is

$$
\begin{array}{r}
U_{\mathrm{eff}}(r)=\frac{1}{\left(E+m_{e} c^{2}\right)}\left[-\frac{2 m_{e} c^{2} a_{s}}{r}+\frac{\hbar^{2} c^{2} l(l+1)+d_{e}}{r^{2}}\right], \\
a_{s}=2 D_{e} r_{e}, \quad d_{s}=\left(E+m_{e} c^{2}\right) D_{e} r_{e}^{2} .
\end{array}
$$

The energy formula is as follows:

$$
\begin{aligned}
& E_{n l} \\
& = \pm m_{e} c^{2}\left[1-\left(\left(4 r_{e}^{2} D_{e}^{2}\right) \times\left(\hbar^{2} c^{2}\right.\right.\right. \\
& \left.\left.\left.\times\left(n_{r}+\sqrt{\left(l+\frac{1}{2}\right)^{2}+\frac{2 r_{e} D_{e}^{2}\left(E+m_{e} c^{2}\right)}{\hbar^{2} c^{2}}}+\frac{1}{2}\right)^{2}\right)^{-1}\right)\right]^{1 / 2},
\end{aligned}
$$

and, the first inspection to the solution in [58], we can obtain the nonrelativistic energy as

$$
E_{n l}^{\prime}=-\frac{8 m_{e} r_{e}^{2} D_{e}^{2}}{\hbar^{2}\left(1+2 n_{r}+\sqrt{(2 l+1)^{2}+\left(8 m_{e} r_{e} D_{e}^{2} / \hbar^{2}\right)}\right)^{2}},
$$

which is identical to (14) of [58].

Fifthly, if potential parameter $a_{s}=0$, the Cornell potential reduces to the relativistic harmonic oscillator potential with $n=2\left(n_{r}+1\right)$. The effective potential is

$$
U_{\mathrm{eff}}(r)=\frac{1}{\left(E+m_{e} c^{2}\right)}\left[2 m_{e} c^{2} b_{s} r+b_{s}^{2} r^{2}+\frac{\hbar^{2} c^{2} l(l+1)}{r^{2}}\right]
$$

which consists of the linear plus harmonic plus centrifugal term potentials. The energy levels can be found as

$$
E_{n l}=m_{e} c^{2}\left[1+\frac{2 \hbar c b_{s}}{m_{e}^{2} c^{4}}\left(2 n_{r}+l+\frac{3}{2}\right)\right]^{1 / 2}
$$


and wave function

$$
\psi_{n, l}(r, \phi)=C_{n, m} \frac{1}{\sqrt{2 \pi}} e^{i m \phi} r^{l+(1 / 2)} e^{-\left(b_{s} / 2 \hbar c\right) r^{2}} \sum_{n=0}^{n_{\max }} a_{n} r^{n},
$$

where

$$
\begin{gathered}
a_{1}=\frac{m_{e} c}{\hbar} a_{0}, \\
a_{2}=-a_{0} \frac{1}{2 \hbar^{2} c^{2}(2 l+(5 / 2))}\left\{E^{2}-m_{e}^{2} c^{4}-\frac{2 \hbar^{3} c^{3}(l+3 / 2)}{b_{s}}\right\} .
\end{gathered}
$$

As before, in the nonrelativistic limit we can also obtain

$$
E^{\prime}=\frac{\hbar b_{s}}{m_{e}}\left(2 n_{r}+l+\frac{3}{2}\right)
$$

Finally, we can take the vector potential of any mixture, say, for example, $a_{v}=0.5 a_{s}$ and $b_{v}=0.5 b_{s}$, then energy equation reads as

$$
\begin{aligned}
E_{n m}= \pm & m_{e}^{2} c^{4}-1.5 a_{s} b_{s} \\
& +2 \hbar c \sqrt{0.75 b_{s}^{2}}\left(\sqrt{\left.m^{2}+\frac{0.75 a_{s}^{2}}{\hbar^{2} c^{2}}+n-1\right)}\right. \\
& \left.-\frac{0.25 a_{s}^{2}\left(E+2 m_{e} c^{2}\right)^{2}}{\hbar^{2} c^{2}\left(n+\sqrt{m^{2}+\left(0.75 a_{s}^{2} / \hbar^{2} c^{2}\right)}-(1 / 2)\right)^{2}}\right]^{1 / 2}
\end{aligned}
$$

and the wave function $\preceq$

$$
\begin{aligned}
& \psi_{n, m}(r, \phi) \\
& =C_{n} \frac{1}{\sqrt{2 \pi}} e^{m \phi} r \sqrt{m^{2}+\left(0.75 a_{s}^{2} / \hbar^{2} c^{2}\right)} \\
& \\
& \times e^{-\left(\sqrt{0.75 b_{s}^{2}} / 2 \hbar c\right) r^{2}-\left(\left(0.5 E+m_{e} c^{2}\right) a_{s} / \hbar^{2} c^{2}\left(n+\sqrt{m^{2}+\left(0.75 a_{s}^{2} / \hbar^{2} c^{2}\right)}-(1 / 2)\right)\right) r} \\
& \quad \times \sum_{n=0}^{n_{\max }} a_{n} r^{n} .
\end{aligned}
$$

\section{Concluding Remarks}

To sum up, in this paper, we used the wave function ansatz method to study the bound state solutions of the KG equation in 2D space for unequal mixture of the Cornell potential with and without external magnetic and $\mathrm{AB}$ flux fields of arbitrary Larmor frequency $\omega_{L} \neq 0$ and $\mathrm{AB}$ strength $\xi \neq 0$. We obtained the energy eigenvalues and wave functions for any arbitrary principal (vibrational) and magnetic (rotational) quantum numbers $n$ and $m$. We applied an appropriate transformation: $m \rightarrow l+(D-1) / 2$ to make our solution valid for any dimension $D$ without magnetic and $A B$ flux fields. The present results show that the problems of relativistic quantum mechanics can be also solved exactly as in the non-relativistic ones.

It is noticed that the solution of $\mathrm{KG}$ with pure scalar Cornell potential provides the general bound state solution for the well-known Cornell plus harmonic oscillator solution for a spinless particle. The bound state solution exists for the relativistic spin-0 particle only when $S(r)>V(r)$. This is obvious from (29) where the KG possesses bound state spectrum when $\left|a_{v}\right| \leq a_{s}$ and $\left|b_{v}\right| \leq b_{s}$ or when the scalar potential is larger than the vector potential there are very few exactly solvable KG equations [59]. The relativistic bound state solutions can also be obtained in this work for any mixture with $V(r)=\beta S(r)$, where $|\beta| \leq 1$. The significance of this bound on $|\beta|$ is the existence of energy eigenvalues (see [60-64] and references therein). The choice of the case $\beta=1$ is simply to reduce our solution to the nonrelativistic (Schrodinger) energy states of the single particle. However, the solution of $\mathrm{KG}$ equation for the case $\beta=-1$ corresponds to the energy states of the antiparticle (antisymmetric case) with some simple transformations in (29); that is, $E \rightarrow$ $-E$ and $V(r) \rightarrow-V(r)$ [39]. The well-known bound state solutions for some special cases including the Coulomb and harmonic oscillator potentials can be recovered without the magnetic and $A B$ flux fields. The results show that the splitting is not constant and mainly dependent on the strength of the external magnetic field and AB flux field. We have seen that the effective potential function and corresponding energy levels are raised in energy when magnetic and $A B$ flux field strengths increase.

The method is effective in solving the singular Cornell potential and produces the desired results. To test the accuracy of our results, specific choices of the potential parameters recover the results of the exactly solvable Coulomb, harmonic oscillator, and Kratzer potentials.

\section{Acknowledgments}

The author thanks the kind referees for their suggestions and comments which have greatly improved the paper. This work is partially supported by the Scientific and Technological Research Council of Turkey.

\section{References}

[1] A. W. Thomas and W. Weise, Structure of the Nucleon, WileyVCH, Berlin, Germany, 2001.

[2] B. Thaller, The Dirac Equation, Springer, New York, NY, USA, 1992.

[3] T.-Y. Wu and W.-Y. P. Hwang, Relativistic Quantum Mechanics and Quantum Fields, World Scientific, Singapore, 1991.

[4] A. S. de Castro, "Klein-Gordon particles in mixed vector-scalar inversely linear potentials," Physics Letters A, vol. 338, no. 2, pp. 81-89, 2005.

[5] S. M. Ikhdair, "Exact Klein-Gordon equation with spatially dependent masses for unequal scalar-vector Coulomb-like 
potentials," European Physical Journal A, vol. 40, no. 2, pp. 143149, 2009.

[6] W.-C. Qiang, R.-S. Zhou, and Y. Gao, "Any l-state solutions of the Klein-Gordon equation with the generalized Hulthén potential," Physics Letters A, vol. 371, no. 3, pp. 201-204, 2007.

[7] S. M. Ikhdair, "Approximate solutions of the dirac equation for the rosen-morse potential including the Spin-orbit centrifugal term," Journal of Mathematical Physics, vol. 51, no. 2, Article ID 023525, 16 pages, 2010.

[8] S. M. Ikhdair, "An approximate $\kappa$ state solutions of the Dirac equation for the generalized Morse potential under spin and pseudospin symmetry," Journal of Mathematical Physics, vol. 52, no. 5, Article ID 052303, 2011.

[9] F. Cooper, A. Khare, and U. Sukhatme, "Supersymmetry and quantum mechanics," Physics Report, vol. 251, no. 5-6, pp. 267385, 1995.

[10] G. Junker, Supersymmetric Methods in Quantum and Statistical Physics, Springer, Berlin, Germany, 1996.

[11] J. M. Fellows and R. A. Smith, "Factorization solution of a family of quantum nonlinear oscillators," Journal of Physics A, vol. 42, no. 33, Article ID 335303, 2009.

[12] R. De, R. Dutt, and U. Sukhatme, "Mapping of shape invariant potentials under point canonical transformations," Journal of Physics A, vol. 25, no. 13, pp. L843-L850, 1992.

[13] N. Kandirmaz and R. Sever, "Coherent states for PT-/non-PTsymmetric and non-Hermitian Morse potentials via the path integral," Physica Scripta, vol. 81, no. 3, Article ID 035302, 2010.

[14] H. Ciftci, R. L. Hall, and N. Saad, "Asymptotic iteration method for eigenvalue problems," Journal of Physics A, vol. 36, no. 47, pp. 11807-11816, 2003.

[15] N. Saad, R. L. Hall, and H. Ciftci, "The Klein-Gordon equation with the Kratzer potential in d dimensions," Central European Journal of Physics, vol. 6, no. 3, pp. 717-729, 2008.

[16] Z.-Q. Ma and B.-W. Xu, "Quantum correction in exact quantization rules," Europhysics Letters, vol. 69, no. 5, pp. 685-691, 2005.

[17] S.-H. Dong and M. Cruz-Irisson, "Energy spectrum for a modified Rosen-Morse potential solved by proper quantization rule and its thermodynamic properties," Journal of Mathematical Chemistry, vol. 50, no. 4, pp. 881-892, 2012.

[18] M. Bag, M. M. Panja, R. Dutt, and Y. P. Varshni, "Modified shifted large- $\mathrm{N}$ approach to the Morse oscillator," Physical Review A, vol. 46, no. 9, pp. 6059-6062, 1992.

[19] S. M. Ikhdair, "Rotational and vibrational diatomic molecule in the Klein-Gordon equation with hyperbolic scalar and vector potentials," International Journal of Modern Physics C, vol. 20, no. 10, pp. 1563-1582, 2009.

[20] S. M. Ikhdair and R. Sever, "A perturbative treatment for the energy levels of neutral atoms," International Journal of Modern Physics A, vol. 21, no. 31, pp. 6465-6476, 2006.

[21] S.-H. Dong, "A new approach to the relativistic schrödinger equation with central potential: Ansatz method," International Journal of Theoretical Physics, vol. 40, no. 2, pp. 559-567, 2001.

[22] S.-H. Dong, "The ansatz method for analyzing Schrödinger's equation with three anharmonic potentials in $D$ dimensions," Foundations of Physics Letters, vol. 15, no. 4, pp. 385-395, 2002.

[23] C. Quigg and J. L. Rosner, "Quantum mechanics with applications to quarkonium," Physics Reports, vol. 56, no. 4, pp. 167-235, 1979.

[24] M. Chaichian and R. Kögerler, "Coupling constants and the nonrelativistic quark model with charmonium potential," Annals of Physics, vol. 124, no. 1, pp. 61-123, 1980.
[25] G. Plante and A. F. Antippa, "Analytic solution of the Schrödinger equation for the Coulomb-plus-linear potential. I. The wave functions," Journal of Mathematical Physics, vol. 46, no. 6, Article ID 062108, 20 pages, 2005.

[26] J. D. Stack, "Heavy-quark potential in SU(3) lattice gauge theory," Physical Review D, vol. 29, no. 6, pp. 1213-1218, 1984.

[27] G. S. Bali, K. Schilling, and A. Wachter, "Complete $O\left(v^{2}\right)$ corrections to the static interquark potential from $\mathrm{SU}(3)$ gauge theory," Physical Review D, vol. 56, no. 5, pp. 2566-2589, 1997.

[28] E. Eichten, K. Gottfried, T. Kinoshita, K. D. Lane, and T.-M. Yan, "Erratum: Charmonium: the model," Physical Review D, vol. 21, no. 1, p. 313, 1980.

[29] E. Eichten, K. Gottfried, T. Kinoshita, K. D. Lane, and T. M. Yan, "Charmonium: comparison with experiment," Physical Review $D$, vol. 21, no. 1, pp. 203-233, 1980.

[30] J.-L. Domenech-Garret and M.-A. Sanchis-Lozano, "Spectroscopy, leptonic decays and the nature of heavy quarkonia," Physics Letters B, vol. 669, no. 1, pp. 52-57, 2008.

[31] J.-L. Domenech-Garret and M.-A. Sanchis-Lozano, "QQ-onia package: a numerical solution to the Schrödinger radial equation for heavy quarkonium," Computer Physics Communications, vol. 180, no. 5, pp. 768-778, 2009.

[32] D. Bessis, E. R. Vrscay, and C. R. Handy, "Hydrogenic atoms in the external potential $V(r)=g r+\lambda r^{2}$ : exact solutions and ground-state eigenvalue bounds using moment methods," Journal of Physics A, vol. 20, no. 2, pp. 419-428, 1987.

[33] Z. Ghalenovi, A. A. Rajabi, and M. Hamzavi, "The heavy baryon masses in variational approach and spin-isospin dependence," Acta Physica Polonica B, vol. 42, no. 8, pp. 1849-1859, 2011.

[34] M. Hamzavi and A. A. Rajabi, "Solution of Dirac equation with Killingbeck potential by using wave function ansatz method under spin symmetry limit," Communications in Theoretical Physics, vol. 55, no. 1, pp. 35-37, 2011.

[35] S.-H. Dong, "On the solutions of the Schrödinger equation with some anharmonic potentials: wave function ansatz," Physica Scripta, vol. 65, no. 4, pp. 289-295, 2002.

[36] M. Znojil, "The generalized continued fractions and potentials of the Lennard-Jones type," Journal of Mathematical Physics, vol. 31, no. 8, pp. 1955-1961, 1990.

[37] S. M. Ikhdair and M. Hamzavi, "Spectral properties of quantum dots influenced by a confining potential model," Physica B, vol. 407, pp. 4797-4803, 2012.

[38] A. A. Rajabi and M. Hamzavi, "Relativistic exect of external magnetic and Aharonov-Bohm fields on the unequal scalar and vector Cornell model," European Physical Journal Plus, vol. 128, pp. 5-6, 2013.

[39] S. M. Ikhdair, "A scalar charged particle in presence of magnetic and Aharonov-Bohm field plus scalar-vector Killingbeck potentials," Few-Body Systems, vol. 54, no. 11, pp. 1987-1995, 2013.

[40] A. Arda and R. Sever, "Effective-mass Klein-Gordon equation for non-PT/non-Hermitian generalized Morse potential," Physica Scripta, vol. 82, no. 6, Article ID 065007, 2010.

[41] M. Hamzavi, S. M. Ikhdair, and K. E. Thylwe, "Pseudospin symmetry in the relativistic Killingbeck potential: quasi-exact solution," Zeitschrift fur Naturforschung A, vol. 67, pp. 567-571, 2012.

[42] S.-H. Dong, Z.-Q. Ma, and G. Esposito, "Exact solutions of the Schrödinger equation with inverse-power potential," Foundations of Physics Letters, vol. 12, no. 5, pp. 465-474, 1999.

[43] S.-H. Dong, "Exact solutions of the two-dimensional Schrödinger equation with certain central potentials," 
International Journal of Theoretical Physics, vol. 39, no. 4, pp. 1119-1128, 2000.

[44] S.-H. Dong, "A new approach to the relativistic schrödinger equation with central potential: Ansatz method," International Journal of Theoretical Physics, vol. 40, no. 2, pp. 559-567, 2001.

[45] W. Greiner, Relativistic Quantum Mechanics: Wave Equations, Springer, Berlin, Germany, 2000.

[46] A. D. Alhaidari, H. Bahlouli, and A. Al-Hasan, "Dirac and Klein-Gordon equations with equal scalar and vector potentials," Physics Letters A, vol. 349, no. 1-4, pp. 87-97, 2006.

[47] R. Khordad, "Effects of magnetic field and geometrical size on the interband light absorption in a quantum pseudodot system," Solid State Sciences, vol. 12, no. 7, pp. 1253-1256, 2010.

[48] R. Khordad, "Simultaneous effects of temperature and pressure on the donor binding energy in a V-groove quantum wire," Superlattices and Microstructures, vol. 47, no. 3, pp. 422-431, 2010.

[49] A. Çetin, "A quantum pseudodot system with a twodimensional pseudoharmonic potential," Physics Letters A, vol. 372, no. 21, pp. 3852-3856, 2008.

[50] S. M. Ikhdair, M. Hamzavi, and R. Sever, "Spectra of cylindrical quantum dots: the effect of electrical and magnetic fields together with AB flux field," Physica B, vol. 407, pp. 4523-4529, 2012.

[51] S. M. Ikhdair and M. Hamzavi, "A quantum pseudodot system with two-dimensional pseudoharmonic oscillator in external magnetic and Aharonov-Bohm fields," Physica B, vol. 407, pp. 4198-4207, 2012.

[52] S. M. Ikhdair and M. Hamzavi, "Effects of extermal fields on a two-dimensional Klein-Gordon particle under pseudoharmonic oscillator interaction," Chinese Physics B, vol. 21, no. 11, Article ID 110302, 2012.

[53] Y. Xu, S. He, and C.-S. Jia, "Approximate analytical solutions of the Klein-Gordon equation with the Pöschl-Teller potential including the centrifugal term," Physica Scripta, vol. 81, no. 4, Article ID 045001, 2010.

[54] R. L. Liboff, Introductory Quantum Mechanics, Addison-Wesley, San Francisco, Calif, USA, 2003.

[55] G. Chen, Z.-D. Chen, and Z.-M. Lou, "Bound states of the KleinGordon and Dirac equation for scalar and vector pseudoharmonic oscillator potentials," Chinese Physics, vol. 13, no. 3, pp. 279-282, 2004.

[56] S. M. Ikhdair and J. Abu-Hasna, "Quantization rule solution to the Hulthén potential in arbitrary dimension with a new approximate scheme for the centrifugal term," Physica Scripta, vol. 83, no. 2, Article ID 025002, 2011.

[57] S. M. Ikhdair and R. Sever, "Exact quantization rule to the Kratzer-type potentials: an application to the diatomic molecules," Journal of Mathematical Chemistry, vol. 45, no. 4, pp. 1137-1152, 2009.

[58] C. Berkdemir, A. Berkdemir, and J. Han, "Bound state solutions of the Schrödinger equation for modified Kratzer's molecular potential," Chemical Physics Letters, vol. 417, no. 4-6, pp. 326329, 2006.

[59] G. Chen, Z.-D. Chen, and P.-C. Xuan, "Semiclassical methods to the Klein-Gordon equation with the unequal scalar and vector potentials," Physica Scripta, vol. 74, no. 3, pp. 367-370, 2006.

[60] S. M. Ikhdair and R. Sever, "Any $l$-state improved quasi-exact analytical solutions of the spatially dependent mass KleinGordon equation for the scalar and vector Hulthén potentials," Physica Scripta, vol. 79, no. 3, Article ID 035002, 2009.
[61] S. M. Ikhdair, "Bound states of the Klein-Gordon for exponential type potentials in D-dimensions," Journal of Quantum Information Science, vol. 1, no. 2, pp. 73-86, 2011.

[62] M. Hamzavi, S. M. Ikhdair, and K. E. Thylwe, "Spinless particles in the field of unequal scalar-vector Yukawa potentials," Chinese Physics B, vol. 22, no. 4, Article ID 040301, 6 pages, 2013.

[63] F. Domínguez-Adame, "Bound states of the Klein-Gordon equation with vector and scalar Hulthén-type potentials," Physics Letters A, vol. 136, no. 4-5, pp. 175-177, 1989.

[64] S. M. Ikhdair and B. J. Falaye, "A charged spinless particles in scalar-vector harmonic oscillators with uniform magnetic and Aharonov-Bohm flux fields," Journal of the Association of Arab Universities for Basic and Applied Sciences. In press. 

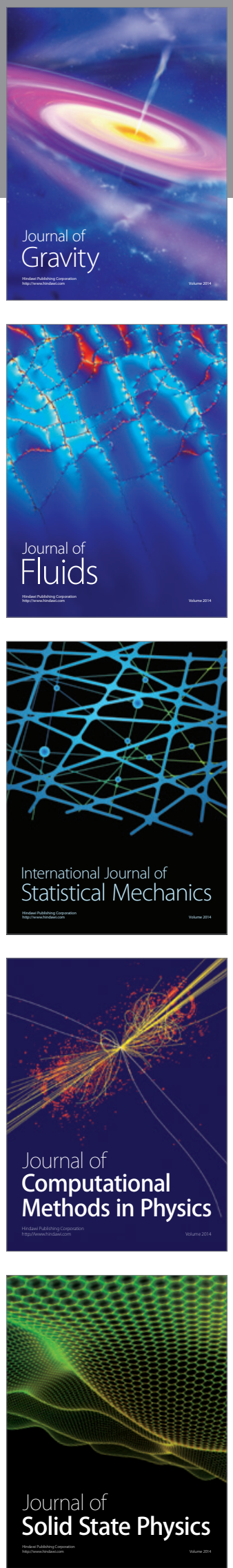

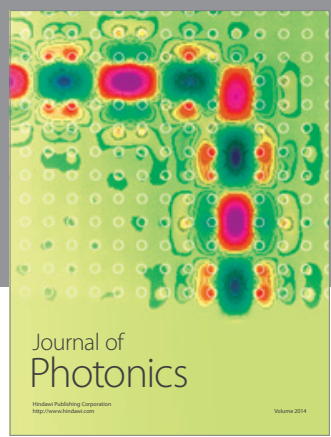

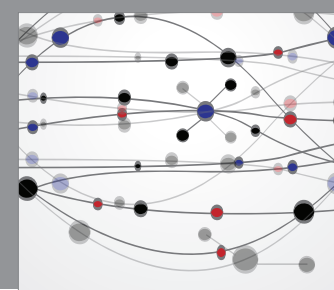

The Scientific World Journal

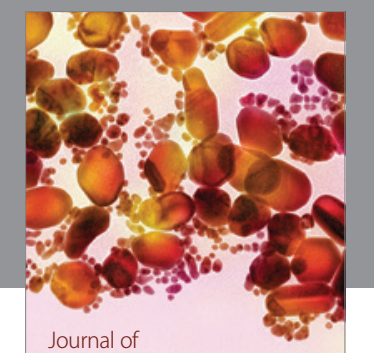

Soft Matter
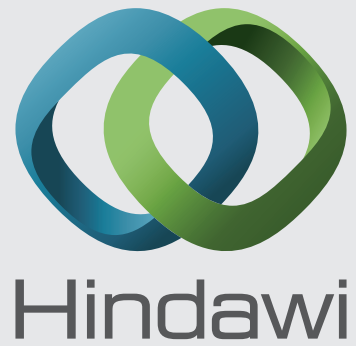

Submit your manuscripts at

http://www.hindawi.com
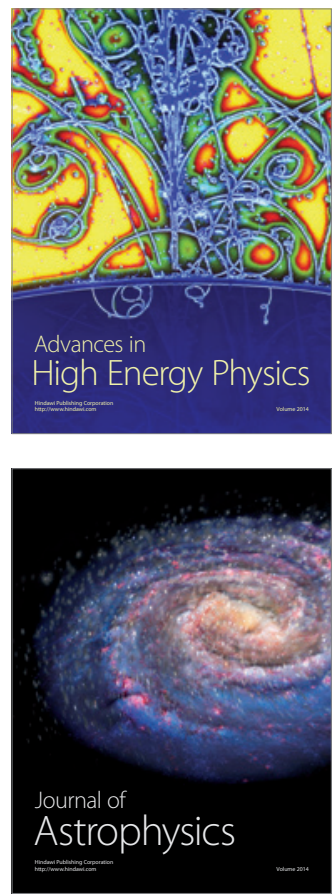
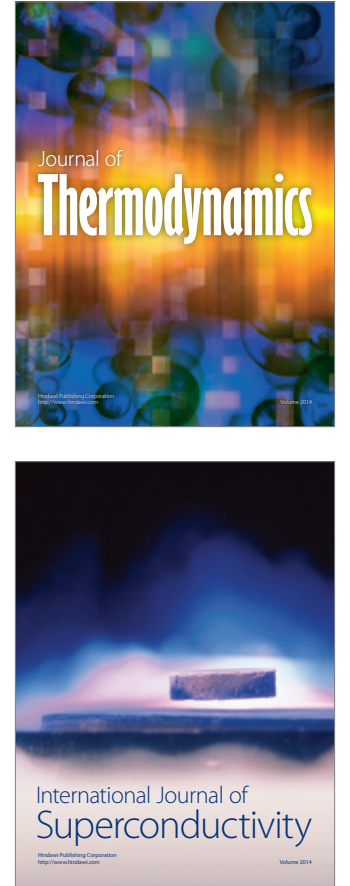
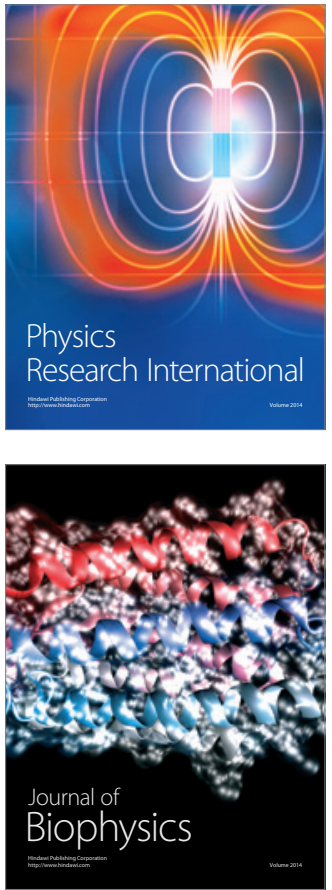
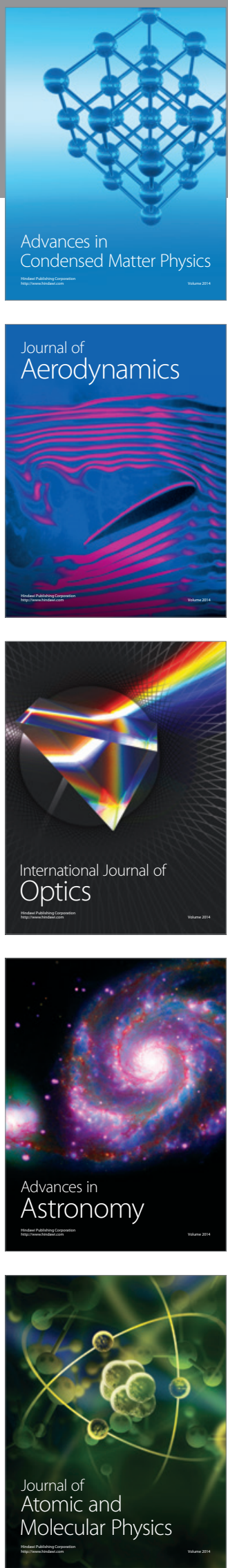